\title{
Planning spatial sampling of the soil from an uncertain reconnaissance variogram
}

\author{
R. Murray Lark ${ }^{1}$, Elliott M. Hamilton ${ }^{1}$, Belinda Kaninga ${ }^{2,3}$, Kakoma K. Maseka ${ }^{4}$, Moola Mutondo ${ }^{4}$, \\ Godfrey M. Sakala ${ }^{2,3}$, and Michael J. Watts ${ }^{1}$ \\ ${ }^{1}$ Centre for Environmental Geochemistry, British Geological Survey, \\ Keyworth, Nottinghamshire, NG12 5GG, UK \\ ${ }^{2}$ Zambia Agriculture Research Institute, Mount Makulu, Central Research Station, Lusaka, Zambia \\ ${ }^{3}$ University of Zambia, Great East Road Campus, Lusaka, Zambia \\ ${ }^{4}$ Copperbelt University, Jambo Drive, Riverside, Kitwe, Zambia
}

Correspondence: R. Murray Lark (mlark@bgs.ac.uk)

\author{
Received: 27 July 2017 - Discussion started: 21 August 2017 \\ Revised: 27 October 2017 - Accepted: 7 November 2017 - Published: 13 December 2017
}

\begin{abstract}
An estimated variogram of a soil property can be used to support a rational choice of sampling intensity for geostatistical mapping. However, it is known that estimated variograms are subject to uncertainty. In this paper we address two practical questions. First, how can we make a robust decision on sampling intensity, given the uncertainty in the variogram? Second, what are the costs incurred in terms of oversampling because of uncertainty in the variogram model used to plan sampling? To achieve this we show how samples of the posterior distribution of variogram parameters, from a computational Bayesian analysis, can be used to characterize the effects of variogram parameter uncertainty on sampling decisions. We show how one can select a sample intensity so that a target value of the kriging variance is not exceeded with some specified probability. This will lead to oversampling, relative to the sampling intensity that would be specified if there were no uncertainty in the variogram parameters. One can estimate the magnitude of this oversampling by treating the tolerable grid spacing for the final sample as a random variable, given the target kriging variance and the posterior sample values. We illustrate these concepts with some data on total uranium content in a relatively sparse sample of soil from agricultural land near mine tailings in the Copperbelt Province of Zambia.
\end{abstract}

\section{Introduction}

When one plans a spatial survey of a soil property by geostatistical mapping, a key choice is the intensity of sampling effort (samples per unit area or, equivalently, spacing of a regular sampling grid). This decision determines the overall cost of sampling but also the precision of the predictions and therefore the uncertainty in the resulting information. It has long been recognized that, when the variogram of the target variable is known, either by reconnaissance sampling or from data in a homologous environment, it is possible to compute the prediction error variances (kriging variance) for sampling grids of different intensity and so to find the amount of sample effort that is required to meet a target level of precision of the spatial predictions (McBratney et al., 1981). This ap- proach has been used in practice (e.g. Di et al., 1989; Ruffo et al., 2005). However, it is often the case that the number of observations available from reconnaissance survey is rather limited, which means that the estimated variogram has considerable uncertainty. This uncertainty in the variogram propagates through the calculation of kriging variance, so the kriging variance achieved by a particular grid spacing has an attendant uncertainty.

Webster and Oliver (1992) suggested that at least 100, and ideally $150-200$, data are required to obtain an adequate estimate of the variogram. This raises a difficulty because for many surveys, 200 data are likely to represent a substantial proportion of the total affordable sampling effort. It may therefore be difficult to justify the collection of this number 
of data for sample planning purposes, except in the case of large surveys on a national scale. An alternative approach was proposed by Marchant and Lark (2006). This is an adaptive sampling strategy in which data are collected in discrete phases. The distribution of sample points for the initial sampling phase is optimized to minimize uncertainty in variogram parameters, given a wide prior distribution for these. In subsequent phases this initial sample is supplemented to improve the precision of the estimates of variogram parameters until the uncertainty in the final sample grid spacing required to complete the survey is reduced to an acceptable size. An advantage of this approach is that the total sample effort is controlled by the evaluation of the quality of information available at the end of each phase, and so the information provided by an initial phase with rather fewer than 100 samples can be exploited. We make no arbitrary assumptions about the requisite sample size. A disadvantage, however, is that it is not always feasible to divide a sampling campaign into several phases, particularly if the region to be surveyed is remote, the samples take a significant time to process and analyse, or the target variable is subject to change over time. Marchant and Lark (2006) suggested that there may, nonetheless, be benefits in simple two-phase optimized designs in which information obtained in the first phase of sampling is used in a numerical optimization procedure to find the distribution of sample points, given those already sampled in phase 1, which minimizes some measure of prediction uncertainty.

These tools for optimization are powerful, but they are computationally costly. Furthermore, they address directly the question of how to deploy some specified number of sample points and are cumbersome if the initial question is what survey intensity is required because this requires a laborious optimization of the distribution of different numbers of sample points. In this paper we consider a simpler approach where the basic procedure of McBratney et al. (1981) is adapted to account for uncertainty in the estimated variogram parameters as quantified in a Bayesian analysis. Such an approach might be useful for making initial decisions on sample effort even if the final distribution of points is optimized using methods such as those of Marchant and Lark (2007).

In this paper we consider a case study from the Copperbelt Province in Zambia. A reconnaissance survey was undertaken on farmland in close proximity to a mine tailings dam. This survey was based on a nested sampling design (Lark et al., 2017), of which we report on 64 samples which were collected at a site of interest, the land farmed by the inhabitants of Mugala village near Kitwe. This sample size is rather fewer than the minimum of 100 suggested by Webster and Oliver (1992).

One variable measured in this survey was the total content of uranium (U) in the soil, and it is that variable which we examine here. Uranium is of interest because the processing of copper ore can produce technically enhanced natu- rally occurring radioactive material (TENORM) in the tailings (residual waste) through the concentration of radionuclides, including uranium. Uranium is known to be a significant constituent of TENORM in wastes produced from copper mines in the Katanga Basin, which includes the Zambian Copperbelt (Katebe et al., 2008).

In this paper we present a Bayesian geostatistical analysis of the data and show how this allows us to quantify the effects of variogram uncertainty on the inferred precision of predictions from sample grids of different spacing and to support a robust choice of sample intensity.

\section{Materials and methods}

\subsection{Field work and data collection}

The study area was approximately 18 ha of farmland used by the inhabitants of Mugala village near Kitwe, located in the Copperbelt Province in the north of Zambia, $\left(12^{\circ} 47^{\prime} 16.1^{\prime \prime} \mathrm{S}\right.$ $28^{\circ} 6^{\prime} 13.2^{\prime \prime}$ E). According to the Exploratory Soil Map of Zambia (1:1000 000), (Ministry of Agriculture, 1991), the soils at and around Kitwe are mapped as plateau soils (legend unit Pu7) which comprises Chromi-haplic Acrisols with Gleyi-haplic Acrisols and partly skeletal phase Dystric Leptosols according to the then-current FAO soil classification (FAO-Unesco, 1974).

A full account of the sampling undertaken in this study is provided by Lark et al. (2017). The sampling was undertaken on transects with sample main stations at intervals of 100 to $200 \mathrm{~m}$. At each main station a soil sample was collected. A second point was collected $100 \mathrm{~m}$ from the main station in a direction approximately normal to the direction of the transect. A third sample point was selected $10 \mathrm{~m}$ from the second in a random direction and a fourth point $1 \mathrm{~m}$ from the third, again in a random direction. The soil samples at each sample point were composites, formed by bulking five cores taken with a Dutch auger $(15 \mathrm{~cm}$ depth of soil from the surface and $5 \mathrm{~cm}$ in diameter). These cores were taken from the corners and centre of a square of approximately $30 \mathrm{~cm}$ length. The samples were placed in paper sample bags, and a few days after collection each sample was reduced in size by coning and quartering. A total of 64 samples were collected this way at Mugala village.

The soil samples were prepared for analysis in the Inorganic Geochemistry laboratories of the British Geological Survey. Each sample was air-dried and then sieved to pass $2 \mathrm{~mm}$. A subsample of the sieved soil was milled to $<53 \mu \mathrm{m}$ in an agate ball mill; then, a $10 \mathrm{~g}$ subsample of milled soil was mixed with $3 \mathrm{~g}$ of binder and a $32 \mathrm{~mm}$ diameter pellet was pressed from this mixture. Total concentrations of a suite of elements were then measured on this pellet by X-ray fluorescence spectrometry (XRFS) and wavelength-dispersive XRFS for U. This analysis was done at the XRFS laboratory of PANalytical Ltd., Nottingham UK. 


\subsection{Data analysis}

\subsubsection{The linear mixed model and its parameters}

Summary statistics were computed for the data, and their spatial distribution was examined. The assumption of a stationary mean seemed plausible from the post plot of the data (Fig. 1b) and the transitive behaviour of the empirical variogram which does not increase at lags longer than about $300 \mathrm{~m}$ (Fig. 2a); so a linear mixed model was proposed for the $n$ data, in which they are treated as a realization of a second-order stationary random variable, $Z$. The model takes the following form:

$z=\mu+\eta+\varepsilon$,

where $\boldsymbol{\mu}$ is a vector, length $n$, with all values equal to a constant mean, and $\boldsymbol{\eta}$ and $\boldsymbol{\varepsilon}$ are mutually independent random effects. The component $\boldsymbol{\eta}$ is distributed as

$\eta \sim \mathcal{N}\left(\mathbf{0}_{n}, \xi \sigma^{2} \mathbf{R}\right)$

and $\boldsymbol{\varepsilon}$ is distributed as

$\boldsymbol{\varepsilon} \sim \mathcal{N}\left(\mathbf{0}_{n},(1-\xi) \sigma^{2} \mathbf{I}_{n}\right)$

where $\mathbf{0}_{n}$ is a vector length $n$ with all elements equal to 0 ; $\mathbf{I}_{n}$ is an $n \times n$ identity matrix; $\mathbf{R}$ is an $n \times n$ correlation matrix; $\sigma$ is the overall variance of $Z$, with $\xi \sigma^{2}$ the variance of the spatially correlated component, $\boldsymbol{\eta}$, and $(1-\xi) \sigma^{2}$ the variance of the independently and identically distributed element, $\boldsymbol{\varepsilon}$. The spatial correlation is modelled under the assumption of second-order stationarity (Webster and Oliver, 2007) such that element $\mathbf{R}[i, j]$ of the correlation matrix can be modelled as a function of the lag interval between the $i$ th and $j$ th observations at locations $s_{i}$ and $s_{j}$ respectively. With a small data set, we assume that the correlation depends only on the length of the lag vector $h=\left|s_{i}-s_{j}\right|$. In this study we used the Matérn correlation function (Matérn, 1986):

$\rho(h)=\left\{2^{\kappa-1} \Gamma(\kappa)\right\}^{-1}\left(\frac{h}{\phi}\right)^{\kappa} K_{\kappa}\left(\frac{h}{\phi}\right)$,

where $\Gamma(\cdot)$ is the gamma function and $K_{\kappa}$ is a modified Bessel function of the second kind of order $\kappa$. The two parameters are $\kappa$, a smoothness parameter, and $\phi$, a distance parameter. These have to be estimated in addition to the variance $\sigma^{2}$ and the parameter $\xi$, which represents the proportion of the variance which is spatially dependent.

Under the linear model with a constant mean the variance parameters $\kappa, \phi, \sigma^{2}$ and $\xi$ can be estimated by maximum likelihood (Lark, 2000; Zimmerman and Stein, 2010; Diggle and Ribeiro, 2007). It is known that the first of these parameters can be difficult to estimate, and Diggle and Ribeiro (2007) suggest that, rather than estimating it along with the other parameters, the marginal likelihood is obtained for a set of discrete values of the parameter (i.e. the likelihood maximized with respect to all the other parameters when $\kappa$ is fixed

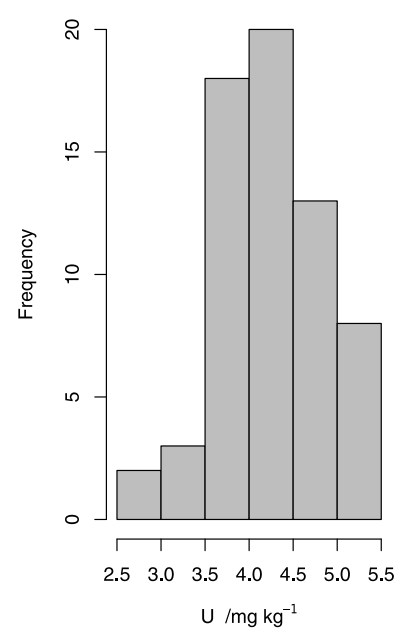

(b)

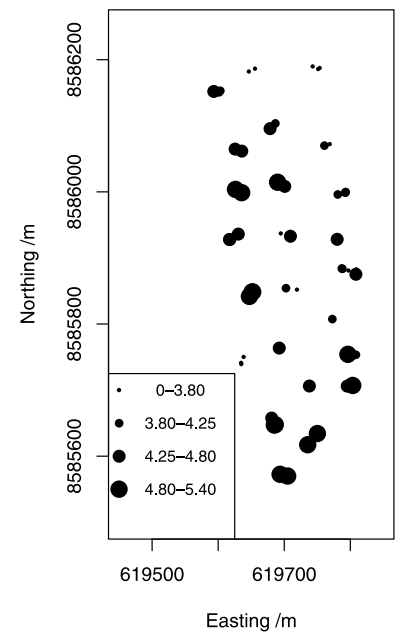

Figure 1. (a) Histogram of uranium concentrations in soil samples and (b) post plot showing the spatial distribution of sample points with symbols indicating concentration intervals delimited by the range and empirical quartiles. The eastings and northings are according to the Universal Transverse Mercator projection zone 35.

at a specified value). Examination of the profile negative loglikelihood function for the $\kappa$ parameter showed that it was potentially troublesome to estimate. Although the negative log likelihood was smaller with $\kappa=2$ than with larger or smaller values, the slope of the marginal likelihood as $\kappa$ was increased above 1.5 was very small. For this reason we followed the guidance of Diggle and Ribeiro (2007) and fixed $\kappa$ at the value for which the profile negative log likelihood was smallest: 2.0. All subsequent analyses are conditional on this choice. Smaller values of $\kappa$ are not implausible, given the shape of the likelihood profile. Selecting 2.0 rather than a smaller value is likely to lead to larger estimates of the uncorrelated "nugget" variance, which will tend to imply larger kriging variances and so is conservative.

Estimates of the other variance parameters were obtained by maximum likelihood. It is possible to quantify uncertainty in these estimates by treating the inverse of the Fisher information matrix as an estimate of the covariance matrix of estimation errors (e.g. Dobson, 1990), but this requires assumptions of linearity which are not plausible for all of the parameters, notably the distance parameter (Marchant and Lark, 2004). An alternative approach is to use a Bayesian formulation of the linear mixed model under which the variance parameters are treated as random variables with a prior distribution, updated to give a posterior distribution by reference to available data. This has been done in previous studies with the linear mixed model applied to soil data (e.g. Orton et al., 2009; Minasny et al., 2011).

A common way to implement the Bayesian analysis is by Markov chain Monte Carlo (MCMC) methods, in which a sample of values from the posterior joint distribution of the 

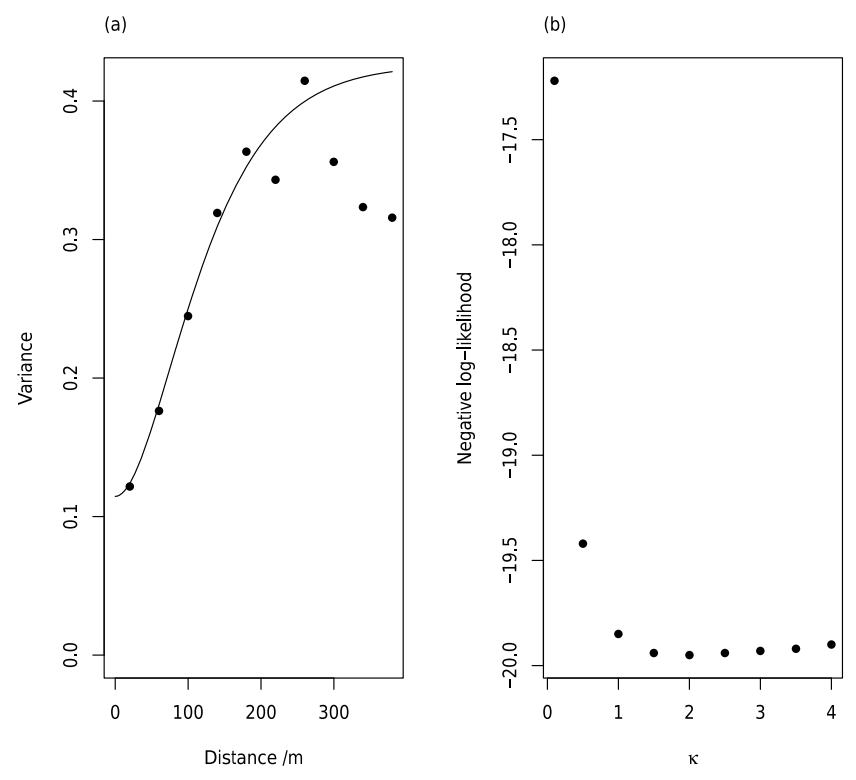

Figure 2. (a) Method-of-moments estimates of the variogram of soil uranium content with the maximum likelihood estimate of the variogram superimposed. (b) Profile values of the negative log likelihood for different values of the $\kappa$ parameter.

variance parameters is drawn. This sample can then be used for further inference about the parameters. Both Orton et al. (2009) and Minasny et al. (2011) used this approach, and we follow the latter in using the "dream" algorithm of Vrugt et al. (2009), as implemented for the R platform ( $\mathrm{R}$ development core team, 2014) by Guillaume and Andrews (2012) for the analysis. For details of this method the reader is referred to Vrugt (2016). In summary, the "dream" algorithm runs multiple MCMC chains in parallel and automatically tunes the "proposal" distribution, which is used to perturb the values in each chain and explore the parameter space so that the resulting sequence of samples has desirable statistical properties. In "dream", and other multichain methods, the perturbation of the chains in each generation is obtained as a combination of a random variate and the difference between the parameters values for randomly selected pairs of chains. In "dream", computational efficiency is achieved by updating only random subsets of the parameters in each generation and by special treatment to identify and manage outlier chains. We used nine chains in our analysis for three parameters, with four chain pairs used to generate the jump at each sample. Other "dream" parameters were set at default values which we have found to be robust over a range of settings. The first $10 \%$ of values in each chain was discarded to avoid the effects of the burn-in period, which is influenced by initial arbitrary settings. Every tenth output of the chain was selected for the final sample to reduce the effects of autocorrelation between samples. The prior distribution for the variogram parameters was uniform over the admissible range $[0,1]$ for $\xi$. For the other parameters the priors were uniform for positive values up to a maximum (10 and 1000 respectively for $\sigma^{2}$ and $\phi$ ). It is important to recall that in a Bayesian analysis all inference is conditional on the priors. In the absence of strong evidence to constrain uniform prior distributions, it is good practice to choose sufficiently wide bounds such that the posterior probability density is negligible near extremes (Diggle and Ribeiro, 2007). On this basis the selected bounds for the prior distributions of $\sigma^{2}$ and $\phi$ were judged to be acceptable.

\subsubsection{Kriging variance as a random variable}

In the analysis undertaken here the smoothness parameter $\kappa$ was fixed at the value selected from the profile likelihood, but the other parameters, $\phi, \sigma^{2}$ and $\xi$, comprise a set which is treated as a random variate:

$\boldsymbol{\Theta}=\left\{\Phi, \Sigma^{2}, \Xi\right\}^{\mathrm{T}}$,

of which we have $m$ MCMC samples:

$\boldsymbol{\theta}_{i}=\left\{\phi_{i}, \sigma_{i}^{2}, \xi_{i}\right\}^{\mathrm{T}}, i=1, \ldots, m$

For some $\boldsymbol{\theta}$, and conditional on a specified interval for a square sample grid, $\lambda$, one can compute the kriging variance at the centre of a grid cell. In this paper we consider the ordinary kriging variance. We may write this kriging variance as a function of $\boldsymbol{\theta}$ :

$v_{\mathrm{k}, \lambda}=f(\boldsymbol{\theta} \mid \lambda)$

In our Bayesian formulation of the problem, the variogram parameters are treated as random variables, and so we may treat the kriging variance, conditional on $\lambda$, as a random variable:

$V_{\mathrm{k}, \lambda}=f(\boldsymbol{\Theta} \mid \lambda)$

We may obtain a sample from this random variable by applying the expression in Eq. (6) to each of the $m$ MCMC samples from $\boldsymbol{\Theta}$ :

$\boldsymbol{v}_{\mathrm{k}, \lambda, i}=f\left(\boldsymbol{\theta}_{i} \mid \lambda,\right), \quad i=1, \ldots, m$.

From this sample we may obtain an estimate of the mean kriging variance, $\bar{v}_{\mathrm{k}, \lambda}$ with the specified grid spacing. We can also compute an empirical estimate of the distribution function for $v_{k, \lambda}$ :

$\widehat{F}(v \mid \lambda)=,\# v_{\mathrm{k}, \lambda}^{\leq v} / m$,

where $\boldsymbol{v}_{\mathrm{k}, \lambda}^{\leq v}$ is the sub-vector comprising all elements of $\boldsymbol{v}_{\mathrm{k}, \lambda}$ which are $\leq v$.

We are planning a spatial sampling exercise and wish to ensure that $v_{\mathrm{k}, \lambda} \leq v_{\mathrm{t}}$, a target kriging variance. Given the uncertainty in the variogram parameters, the kriging variance 
achieved by some grid spacing, $\lambda$, is uncertain. One way to deal with this is to ensure that the probability that the target kriging variance is not exceeded for a chosen spacing is sufficiently large. This is analogous to power analysis for hypothesis testing. If we specify a probability of, for example, 0.8 , then we could select a grid spacing, $\breve{\lambda}$ such that

$\widehat{F}\left(v_{\mathrm{t}} \mid \breve{\lambda}\right)=0.8$.

We undertook these calculations using the sample of variogram parameters obtained from the MCMC chains as described in the previous section. The kriging variance for each sample was computed for the cell centre of square sample grids considering spacings up to $300 \mathrm{~m}$.

The target kriging variance, $v_{\mathrm{t}}$, might be selected on various grounds (de Gruijter et al., 2006; Black et al., 2008; Lark and Knights, 2015). For illustrative purposes we chose to select a target kriging variance such that the kriging standard error was $10 \%$ of the overall mean concentration. This gives a target kriging variance of $v_{\mathrm{t}}=0.18$ in this case.

We computed, for each of a set of grid spacings up to $300 \mathrm{~m}$, the estimate of $\widehat{F}\left(v_{\mathrm{t}} \mid \lambda\right)$ and identified the spacing at which this value was 0.8 .

\subsubsection{Tolerable grid spacing as a random variable}

The previous section shows how one may select a grid spacing such that the probability that the kriging variance at the centre of a grid cell is less than or equal to a target variance is sufficiently large. We may then wish to quantify the implications of the uncertainty in terms of the extent to which we are likely to have oversampled to account for it. To do this we introduce the notion of the tolerable grid spacing as a random variable given uncertainty in the variogram parameters.

For some sample from the variogram parameters, $\boldsymbol{\theta}$, it may be possible to find $\lambda_{v}$, the spacing of a square grid such that the kriging variance at the centre of a grid cell is $v$. This is done numerically, by interpolation between evaluations of the kriging variance for a series of grid spacings and the particular parameter set using Eq. (6). The value $\lambda_{v}$ is not necessarily defined for some parameter set, $\boldsymbol{\theta}$. There are two circumstances in which it is not. The first is when

$v>\sigma^{2}+\psi_{\lambda}, \forall \lambda$,

where $\psi_{\lambda}$ denotes the Lagrange parameter of the ordinary kriging estimate at the centre of a grid cell of spacing $\lambda$. In this circumstance the target kriging variance is so large that it is matched by kriging from the coarsest grid. The second circumstance in which $\lambda_{v}$ is not defined is when the spatially uncorrelated variance is larger than $v$ :

$v<\xi \sigma^{2}$.
In this latter case the kriging variance $v$ cannot be achieved at the centre of the grid cell however fine the spacing. When

$\xi \sigma^{2}<v<\sigma^{2}+\psi_{\lambda} \forall \lambda$,

then a unique value of $\lambda_{v}$ is defined for the $\boldsymbol{\theta}$ and $v$ under consideration, assuming ordinary point kriging or block kriging for a block which is small compared with the grid of observations from which the prediction is made. When $v$ takes a value in the range for which $\lambda_{v}$ is defined, we may express the relationship by a function:

$\lambda_{v}=g(v, \boldsymbol{\theta})$.

We call $\lambda_{v}$ the tolerable grid spacing because it is the coarsest spacing compatible with the kriging variance $v$. In the Bayesian approach, where $\boldsymbol{\theta}$ is drawn from a random variable $\Theta$, we can treat the tolerable grid spacing as a random variable $\Lambda_{v}$ :

$\Lambda_{v}=g(v, \boldsymbol{\Theta})$.

Consider a situation in which an oracle provides variogram parameters without uncertainty and we can therefore compute the tolerable grid spacing for target variance $v_{\mathrm{t}}$ without uncertainty. The sample density is $\lambda_{v_{\mathrm{t}}}^{-2}$ samples per unit area. If, in the same circumstances, we did not have access to the oracle but selected a grid spacing, $\breve{\lambda}$, to achieve Eq. (10), then the oversampling, attributable to our strategy to manage uncertainty would be $\breve{\lambda}^{-2}-\lambda_{v_{\mathrm{t}}}^{-2}$ samples per unit area. This could be directly translated to a cost given analytical costs and logistical costs (e.g. Lark and Knights, 2015). In practice we may use our $m$ MCMC samples of the variogram parameters, $\boldsymbol{\theta}_{i}$, to compute a set of tolerable grid spacings, where this is defined as

$$
\begin{aligned}
& \lambda_{v_{\mathrm{t}}, \lambda, i}=g\left(v_{\mathrm{t}}, \boldsymbol{\theta}_{i}\right) ; \text { if } \xi_{i} \sigma_{i}^{2}<v<\sigma_{i}^{2}+\psi_{\lambda} \forall \lambda ; \\
& \quad i=1, \ldots, m .
\end{aligned}
$$

One may note the proportion of the MCMC samples for which the tolerable grid spacing is defined. If the numbers of undefined cases are significant, then one should note whether this is because the target variance is commonly large relative to the variance $\sigma^{2}$, as in Eq. (11), or small relative to the uncorrelated variance $\xi \sigma^{2}$, as in Eq. (12). Either would suggest that a review of the target variance is needed. Otherwise one may compute the corresponding sample densities for each variate in the sample of tolerable grid spacing and compare these with the sample density used to achieve the condition in Eq. (10). The difference is the oversampling, which, as noted above, can be converted to a cost. If this cost is large, then there are two options. The first is to accept a larger target variance or to leave the target variance unchanged but to accept a smaller probability that it is achieved. The second is to consider further exploratory sampling to reduce the uncertainty in the variogram parameters. The latter approach may 
Table 1. Summary statistics on soil uranium content.

\begin{tabular}{lr}
\hline$n$ & 64 \\
Mean $\left(\mathrm{mg} \mathrm{kg}^{-1}\right)$ & 4.29 \\
Median $\left(\mathrm{mg} \mathrm{kg}^{-1}\right)$ & 4.25 \\
Standard deviation $\left(\mathrm{mg} \mathrm{kg}^{-1}\right)$ & 0.60 \\
Skewness & -0.13 \\
\hline
\end{tabular}

Table 2. Maximum likelihood estimate of spatial covariance parameters for soil uranium.

\begin{tabular}{lr}
\hline$\phi(\mathrm{m})$ & 55.50 \\
$\kappa^{*}$ & 2 \\
$\sigma^{2}$ & 0.43 \\
$\xi$ & 0.73 \\
\hline \multirow{2}{*}{ Obtained by profile } \\
likelihood.
\end{tabular}

be attractive when a survey is planned over a large region, and additional sampling effort is small relative to the total sample effort expected.

We followed these procedures, using Eq. (15) to compute a sample of tolerable grid spacings for the target kriging variance of 0.18 . We noted the proportion of samples for which this tolerable grid spacing was defined. We computed the corresponding values of the sample density.

We then repeated these calculations assuming that (i) the target kriging variance is 0.18 but that we are prepared to accept a smaller probability that it is not exceeded $(0.75$ or 0.70 ) and (ii) that we can accept a larger target kriging variance of 0.25 but require a probability of 0.8 that it is not exceeded.

\section{Results}

Table 1 shows summary statistics for the data on uranium, and their histogram is shown in Fig. 1a. The data are reasonably symmetrically distributed, and there is no evidence from these data that a transformation is required. The empirical variogram (solid symbols in Fig. 2a) shows no evidence of a spatial trend. The profile negative log likelihoods for different values of the $\kappa$ parameter are shown in Fig. $2 b$. On the basis of these results $\kappa$ was fixed at 2.0. The maximum likelihood estimates of the parameters are presented in Table 2, and the solid line in Fig. 2a shows the corresponding variogram.

Figure 3 shows the empirical posterior distributions of the three variogram parameters (conditional on fixing $\kappa=2$ ) corresponding to 15282 samples after removing the first $10 \%$ for burn-in and then extracting every tenth. Note that the empirical density is small near the upper prior limit for $\phi(1000 \mathrm{~m})$ and for $\sigma^{2}(10)$. The horizontal bars show the $95 \%$ credible intervals for each parameter. Because the distributions are not symmetrical, the credible intervals were the
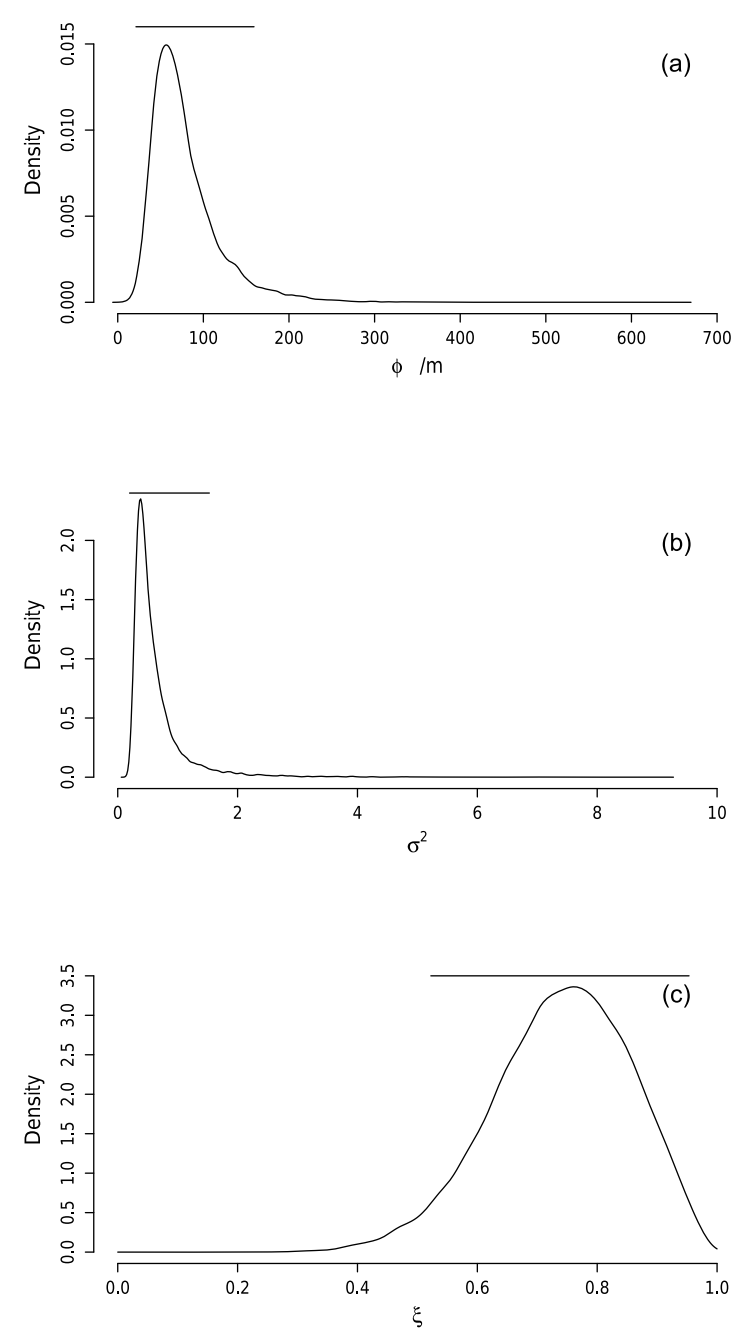

Figure 3. Empirical probability density functions for variogram parameters: $\phi(\mathbf{a}), \sigma^{2}$ (b) and $\xi(\mathbf{c})$. These were obtained from the MCMC samples. Horizontal bars show the $95 \%$ credible interval for each parameter.

highest density intervals (i.e. for a unimodal distribution the narrowest interval over which the integral of the probability density is 0.95 ). This was computed using the "hdi" procedure from the "HDInterval" package for the R platform (Meredith and Kruschke, 2016).

Figure 4 shows the probability, estimated from the MCMC samples, that the kriging variance at the centre of a square grid of spacing $\lambda \mathrm{m}$, will be less than or equal to the target kriging variance 0.18 . The graph shows the spacing $(44 \mathrm{~m}$; 5.12 samples ha ${ }^{-1}$ ), at which this probability is 0.8 . The grid spacing at which the expected kriging variance is equal to the target can be identified from the graph in Fig. 5 (solid line); the grid spacing is $75 \mathrm{~m}$ (1.78 samples ha-1). If one considers only the maximum likelihood estimates of the variogram parameters (Table 2), then the relationship between grid spacing and kriging variance is given by the dashed line 


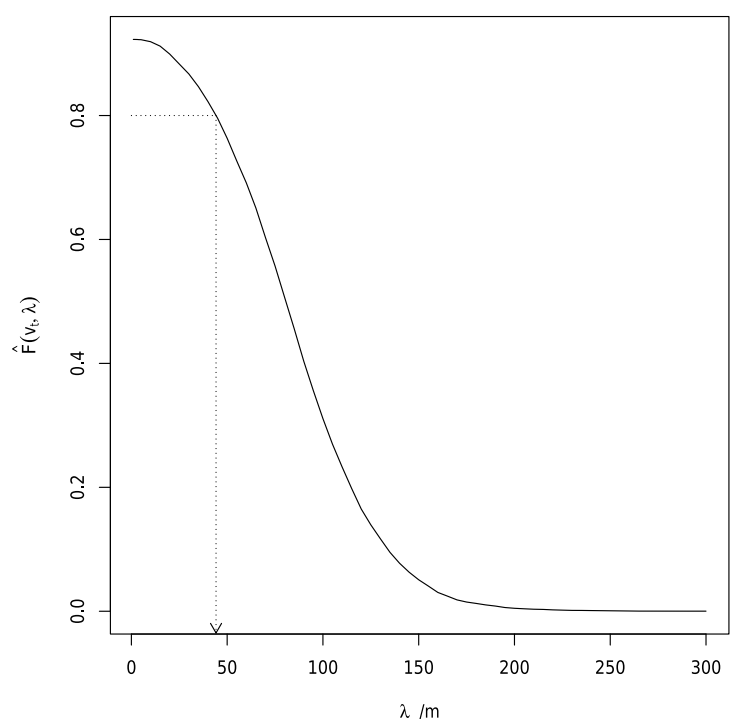

Figure 4. Estimates of $\widehat{F}\left(v_{\mathrm{t}} \mid \lambda\right.$, ) (Eq. 9) for different grid spacings with $v_{\mathrm{t}}$ set to 0.18 . The dotted line shows the grid spacing $(44.2 \mathrm{~m})$ at which $\widehat{F}\left(v_{\mathrm{t}} \mid \lambda,\right)=0.8$.

in Fig. 5, and the target kriging variance is equivalent to a grid spacing of $85 \mathrm{~m}\left(1.38\right.$ samples ha $\left.^{-1}\right)$.

Treating the grid spacing which achieves the target kriging variance of 0.18 as a random variable, $\Lambda_{v_{\mathrm{t}}}$ (tolerable grid spacing), we found that this was defined for $99.22 \%$ of the MCMC samples. Figure 6a shows the empirical probability density function (PDF) of the grid spacings, $\lambda_{v_{t}}$. This distribution is mildly positively skewed with some values in an upper tail, but the mean $(86.4 \mathrm{~m})$ and median $(84.4 \mathrm{~m})$ are very close. The distribution of corresponding sample densities (rescaled to samples per ha) is strongly skewed and is shown on a log scale in Fig. 5b. The median tolerable sample spacing is 1.4 samples $\mathrm{ha}^{-1}$, the mean is strongly influenced by the upper tail, and its value ( 5.32 samples ha $^{-1}$ ) is the 87 th empirical percentile of the observations. For this reason we summarize the distribution of tolerable sample densities by its median.

We can now summarize the practical implications of this analysis for further sampling of the soil to map uranium in this environment or comparable ones. The variogram is uncertain, which is not surprising given the relatively small sample size. Because of this, for any specified sample grid spacing, we cannot be sure about the expected prediction error variance at the centre of a grid cell because this depends on variogram parameters about which we are uncertain. If a decision on sample density were based on the empirical variogram parameters alone, with no attention to their uncertainty, then these results show that the sample effort required to ensure that prediction error variances are below some threshold may be markedly underestimated.

On the basis of our Bayesian analysis we can characterize the uncertainty in the variogram parameters, and so we can

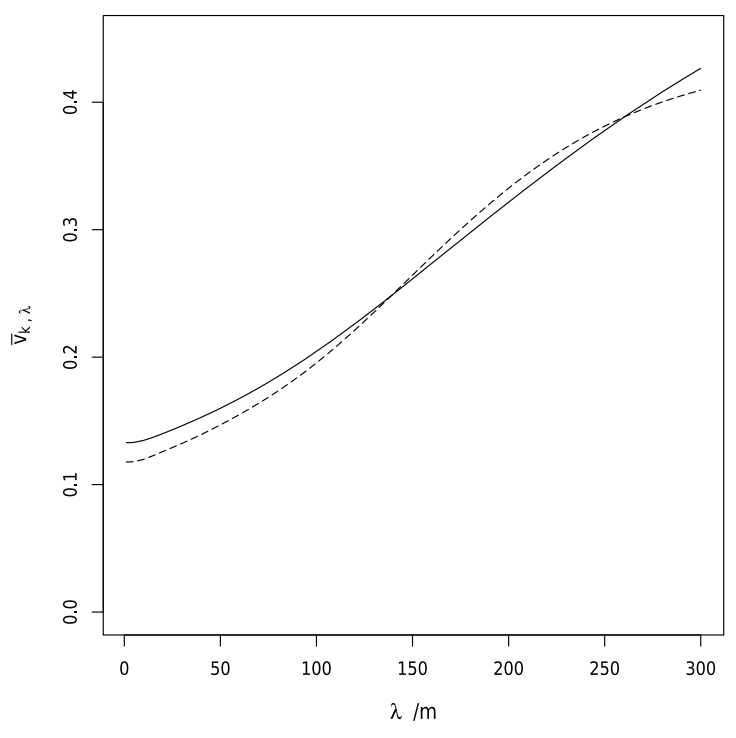

Figure 5. Value of kriging variance at the centre of a square grid cell as a function of grid spacing, $\lambda$. The solid line is the expected value of kriging variance over the distribution of parameters, and the dashed line are the values given the maximum likelihood estimates of those parameters.
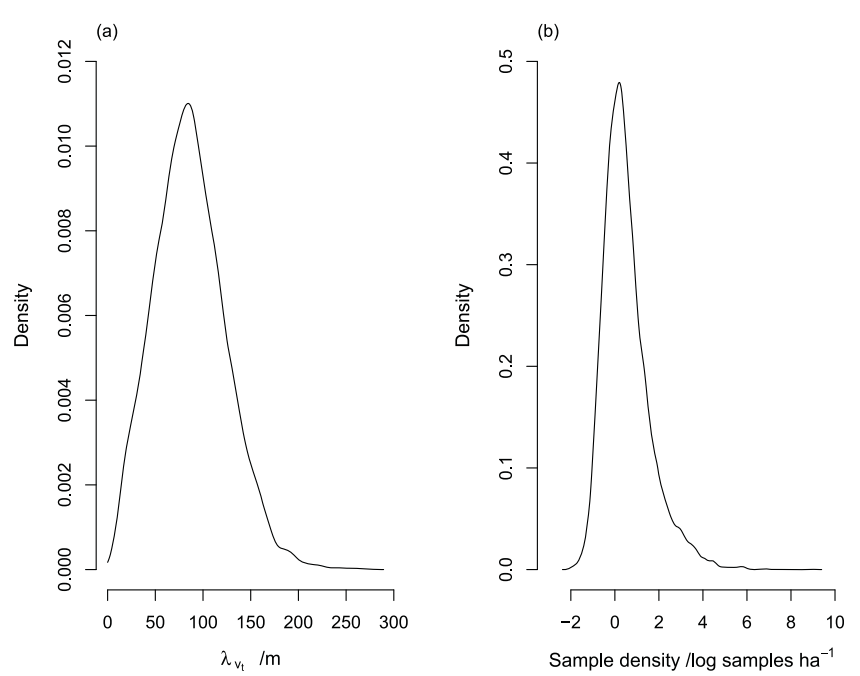

Figure 6. Empirical probability density function for grid spacing that achieves the target kriging variance $(0.18)$ and corresponding PDF for the logarithm of the sample density which achieves the same.

compute the probability that the kriging variance for some specified grid cell is below our maximum acceptable value. A strategy to deal with the uncertainty about the variogram parameters is to find a grid spacing such that the probability that our target kriging variance is not exceeded is judged to be sufficient. In this case we find that selecting a sample density of 5.12 samples ha $^{-1}$ ensures that the probability the specified kriging variance is not exceeded is 0.8 . 
The achievement of this level of confidence in the quality of our final spatial predictions comes with a cost; we have to do more sampling than we would if the variogram parameters were known with greater certainty. Our analysis shows that the median-unbiased tolerable grid spacing for the target variance is 1.4 samples ha $^{-1}$ (close to what we would have obtained if we used the maximum likelihood estimate of the variogram parameters to find the grid spacing). This means that the median-unbiased oversampling due to the uncertainty is 3.72 samples ha ${ }^{-1}$. This could be a substantial cost for a survey of a large region.

If this cost is unacceptable, then there are three approaches we could take. The first is to allow greater uncertainty that our final predictions are of the target precision. We considered the effect of basing the sampling decision on a smaller probability that the target kriging variance is not exceeded, with the target kriging variance held at 0.18 . If we specify a probability of 0.75 rather than 0.8 , then the grid spacing increases to $53 \mathrm{~m}$ (sample density of 3.54 per ha) and the median-unbiased oversampling due to uncertainty is reduced to 2.1 per ha. Reducing the probability further to 0.7 , the grid spacing is $56 \mathrm{~m}$, and the sample density is 3.13 per ha, with a median-unbiased over sampling of 1.7 per ha.

A second approach is to accept a larger target kriging variance. We considered a value of 0.25 . The probability that this is not exceeded is 0.8 with a grid spacing of $110 \mathrm{~m}(0.82 \mathrm{sam}-$ ples $\mathrm{ha}^{-1}$ ), and the median-unbiased oversampling in this case is 0.38 samples ha ${ }^{-1}$ (the tolerable grid spacing was defined for $97 \%$ of cases because of the increased probability that the kriging variance would be bounded at a smaller value than the target).

Both these approaches require that we tolerate greater uncertainty, either in the final predictions (accepting a larger kriging variance) or our level of confidence that the specified kriging variance is achieved. If neither of these is acceptable, then we must collect additional data to reduce the uncertainty in the variogram model before planning the final survey.

\section{Conclusions}

In this paper we have introduced two new concepts for use in the planning of a geostatistical survey. In the first we consider the kriging variance achieved with a particular grid spacing as a random variable, given a set of MCMC samples of the variogram parameters. This allows us to select a grid spacing such that the probability that the target kriging variance is not exceeded is met with a specified probability. The second concept is to treat the tolerable grid spacing as a random variable. This allows us to quantify the effects of variogram uncertainty in terms of expected oversampling. These provide a framework for decision-making about geostatistical sampling based on an uncertain variogram. We have shown that, for some plausible values of the target kriging variance, and required probability that the kriging variance is not exceeded, it may be necessary to accept that considerable oversampling is necessary. This can be reduced by changing either condition. The scientist planning sampling, or stakeholders such as regulator or policy makers, can explore the effect of relaxing either the target kriging variance or the probability that it is not exceeded.

An alternative, if the expected oversampling is large, is to put some additional sampling effort into improving the quality of the variogram estimate on which the final survey is to be based. Within the Bayesian framework, we cannot compute an expected effect on the parameter uncertainty of including some specified number of additional sample points. However, we could optimize the distribution of the additional points using the procedures of Marchant and Lark (2006), and if the amount of additional sampling is limited to, for example, $10 \%$ of the median-unbiased estimate of oversampling incurred with the uncertainty in the current variogram, that will ensure that additional sampling to improve the variogram is not excessive.

There is scope for further work on the methods presented in this paper. First, we note that the study is based on ordinary kriging, in which the mean is assumed to be stationary. The method could be extended to more general cases of the empirical best linear unbiased predictor (e.g. universal kriging). In this case, however, the prediction error variance has a component due to uncertainty in the parameters which describe the non-stationary mean (e.g. coefficients of a trend surface). The computed prediction error variance for different sample strategies will therefore be conditional on assumptions about the model for the mean (e.g. that it is a second-order polynomial in the rectilinear coordinates). The approach could be extended to encompass other sources of uncertainty in the variogram model (e.g. measurement or location error) or the use of a variogram estimated from geophysical data which can be regarded only as a proxy for the target variable, provided that these uncertainties can be incorporated into a Bayesian formulation.

Second, in this study we considered a uniform sample grid, which is appropriate for sampling a relatively large and uniform region. It would be straightforward to do the same computations on sample designs obtained for non-uniform regions by spatial coverage methods such as those presented in the "spcova" package for the R platform (Walvoort et al., 2010).

In conclusion, our example shows that uncertainty in an exploratory variogram can have significant implications in terms of sampling cost under reasonable rules to guide sample planning (in terms of the target kriging variance and the probability one requires that the target kriging variance is not exceeded under the final sampling design). A corollary of this is that sample planning from an empirical variogram which does not take account of its uncertainty will often fail to achieve efficient and adequate sample designs. The concepts introduced in this paper allow a rational assessment of whether currently available data suffice to support a decision 
on detailed sampling for mapping or whether additional exploratory sampling is needed before a final survey design is fixed. In summary, the practical guidelines for sample planning are as follows.

1. Identify a target kriging variance which represents an acceptable precision for the final spatial predictions of the soil property of interest.

2. Decide on a level of confidence, expressed in terms of a probability that the target precision is achieved, which is required for sample planning.

3. By a Bayesian analysis of available data, obtain samples from the posterior distributions of variogram properties, and from these identify the sample density required to achieve the target precision determined at step (1) with the probability determined at step (2).

4. Find the distribution of tolerable grid spacings, given the sample of variogram parameters, and from this compute the oversampling required to achieve the target precision with acceptable probability.

5. If this level of oversampling is unacceptable, then either

i. review the decisions made at steps (1) and (2), increasing the acceptable kriging variance or reducing the acceptable probability of achieving this or both.

ii. plan additional sampling to improve the estimate of the variogram. The procedures presented by Marchant and Lark (2006) may be used to plan this additional sampling, but we cannot compute the required additional sampling in the Bayesian framework. The amount of oversampling expected with current levels of uncertainty might provide a basis for deciding how much additional sampling to undertake at the reconnaissance stage.

Data availability. The original data are available as a Supplement to the online version of this paper, "Mugala_U.txt". This is an ASCII file, each row corresponding to a sample site. The first two columns are eastings and northings (units of metres; Universal Transverse Mercator projection zone 35. The third column is the total $\mathrm{U}$ content in $\mathrm{mg} \mathrm{kg}^{-1}$.

\section{The Supplement related to this article is available online at https://doi.org/10.5194/soil-3-235-2017-supplement.}

Competing interests. The authors declare that they have no conflict of interest.
Acknowledgements. This paper is published with permission of the Executive Director of the British Geological Survey (Natural Environment Research Council). Field and laboratory work for this project was funded by The Centre for Environmental Geochemistry and BGS Global. Author contributions were supported by the UK Department for International Development (DFID) through a Royal Society and DFID Africa Capacity Building Initiative (ACBI) Programme Grant, Award AQ140000. We acknowledge the assistance of staff from ZARI and students at Copperbelt University for help with field sampling. We are grateful to the people of Mugala village for permission to sample on their fields.

Edited by: Paul Hallett

Reviewed by: David Rossiter and one anonymous referee

\section{References}

Black, H., Bellamy, P., Creamer, R., Elston, D., Emmett, B., Frogbrook, Z., Hudson, G., Jordan, C., Lark, M. Lilly, A., Marchant, B., Plum, S., Potts, J., Reynolds, B., Thompson, P., and Booth, P.: Design and operation of a UK soil monitoring network, Environment Agency, Bristol, Science Report - SC060073, 2008.

de Gruijter, J. J., Brus, D. J., Biekens, M. F. P., and Knotters, M.: Sampling for Natural Resource Monitoring, Springer, Berlin, 2006.

Di, H. J., Trangmar, B. B., and Kemp, R. A.: Use of geostatistics in designing sampling strategies for soil survey, Soil Sci. Soci. Am. J., 53, 1163-1167, 1989.

Diggle, P. J. and Ribeiro, P. J.: Model-Based Geostatistics, Springer, New York, 2007.

Dobson, A. J.: An Introduction to Generalized Linear Models, Chapman \& Hall, London, 1990.

FAO-Unesco: Soil Map of the World, 1 : 5000 000, Volume 1, Legend, Unesco, Paris, 1974.

Guillaume, J. and Andrews, F.: dream: DiffeRential Evolution Adaptive Metropolis, R package version 0.4-2., available at: http: //dream.r-forge.r-project.org/ (last access: 8 December 2017), 2012.

Katebe, R., Michalik, B., Phiri, Z., and Nkhuwa, D. C. W.: Status of naturally occurring radionuclides in copper mine wastewater in Zambia, in: Proceedings of the Fifth International Symposium on Naturally Occurring Radioactive Material, International Atomic Energy Agency, Vienna, 409-417, 2008.

Lark, R. M.: Estimating variograms of soil properties by the method-of-moments and maximum likelihood; a comparison, Eur. J. Soil Sci., 51, 717-728, 2000.

Lark, R. M. and Knights, K. V.: The implicit loss function for errors in soil information, Geoderma, 251-252, 24-32, 2015.

Lark, R. M., Hamilton, E. M., Kaninga, B., Maseka, K. K., Mutondo, M., Sakala, G. M., and Watts, M. J.: Nested sampling and spatial analysis for reconnaissance investigations of soil: an example from agricultural land near mine tailings in Zambia, Eur. J. Soil Sci., 68, 605-620, 2017.

Marchant, B. P. and Lark, R. M.: Estimating variogram uncertainty, Math. Geol., 36, 867-898, 2004.

Marchant, B. P. and Lark, R. M.: Adaptive sampling for reconnaissance surveys for geostatistical mapping of the soil, Eur. J. Soil Sci., 57, 831-845, 2006. 
Marchant, B. P. and Lark, R. M.: Optimized sample schemes for geostatistical surveys, Math. Geol., 39, 113-134, 2007.

Matérn, B.: Spatial Variation, Lecture Notes in Statistics, No. 36, Springer, New York, 1986.

McBratney, A. B., Webster, R., and Burgess, T. M.: The design of optimal sampling schemes for local estimation and mapping of regionalised variables. I. Theory and Method, Comput. Geosci., 7, 331-334, 1981.

Meredith, M. and Kruschke, J.: HDInterval: Highest (Posterior) Density Intervals, R package version 0.1.3., available at: https:// cran.r-project.org/web/packages/HDInterval/index.html (last access: 8 December 2017), 2016.

Minasny, B., Vrugt, J. A., and McBratney, A. B.: Confronting uncertainty in model-based geostatistics using Markov Chain Monte Carlo simulation, Geoderma, 163, 150-162, 2011.

Ministry of Agriculture: Exploratory Soil Map of Zambia, Scale $1: 1.000 .000$, Ministry of Agriculture, Lusaka, 1991.

Orton, T. G., Rawlins, B. G., and Lark, R. M.: Using measurements close to a detection limit in a geostatistical case study to predict selenium concentration in topsoil, Geoderma, 152, 269-282, 2009.

R Development Core Team: R: A language and environment for statistical computing, R Foundation for Statistical Computing, Vienna, Austria, available at: http://www.R-project.org/ (last access: 8 December 2017), 2014.
Ruffo, M. L., Bollero, G. A., Hoeft, R. G., and Bullock, D. G.: Spatial variability of the Illinois soil nitrogen test: implications for soil sampling, Agron. J., 97, 1485-1492, 2005.

Vrugt, J. A.: Markov chain Monte Carlo simulation using the DREAM software package: theory, concepts and MATLAB implementation, Environ. Model. Softw., 75, 273-316, 2016.

Vrugt, J. A., Ter Braak, C. J. F., Diks, C. G. H., Robinson, B. A., Hyman, J. M., and Higdon, D.: Accelerating Markov chain Monte Carlo simulation by differential evolution with self-adaptive randomized subspace sampling, Int. J. Nonlin. Sci. Num., 10, 273 290, 2009.

Walvoort, D. J. J., Brus, D. J., and de Gruijter, J. J.: An R package for spatial-coverage sampling and random sampling from compact geographical strata by k-means, Comput. Geosci., 36, 12611267, 2010.

Webster, R. and Oliver, M. A.: Sample adequately to estimate variograms of soil properties, J. Soil Sci., 43, 177-192, 1992.

Zimmerman, D. L. and Stein, M.: Classical geostatistical methods, in: Handbook of Spatial Statistics, edited by: Gelfand, A. E., Diggle, P. J., Fuentes, M., and Guttorp, P., CRC Press, Boca Raton, 29-44, 2010. 